\title{
A!
}

This is an electronic reprint of the original article.

This reprint may differ from the original in pagination and typographic detail.

Haghighat Khajavi, Siavash; Jaribion, Alireza; Knapen, Adriaan; Abiedat, Leila

\section{Digital Twin for Safety and Comfort: A Case Study of Sauna}

Published in:

Proceedings - IECON 2020

DOI:

10.1109/IECON43393.2020.9254270

Published: 18/10/2020

Document Version

Peer reviewed version

Please cite the original version:

Haghighat Khajavi, S., Jaribion, A., Knapen, A., \& Abiedat, L. (2020). Digital Twin for Safety and Comfort: A

Case Study of Sauna. In Proceedings - IECON 2020: 46th Annual Conference of the IEEE Industrial Electronics Society (pp. 167-172). [9254270] IEEE. https://doi.org/10.1109/IECON43393.2020.9254270

This material is protected by copyright and other intellectual property rights, and duplication or sale of all or part of any of the repository collections is not permitted, except that material may be duplicated by you for your research use or educational purposes in electronic or print form. You must obtain permission for any other use. Electronic or print copies may not be offered, whether for sale or otherwise to anyone who is not an authorised user. 


\title{
Digital Twin for Safety and Comfort: A Case Study of Sauna
}

\author{
Siavash H. Khajavi $\dagger$, Alireza Jaribion $\dagger$, Adriaan Knapen $\ddagger$, and Leila Abiedat $\dagger \dagger$ \\ $\dagger$ Department of Industrial Engineering and Management, Aalto University, Finland \\ $\ddagger$ Department of Computer Science,Aalto University, Finland \\ $\dagger †$ Department of Sociology, St. Petersburg State University, Russia \\ $\dagger, \ddagger$ Emails: firstname.lastname@aalto.fi, $\dagger \dagger$ leila.abiedat@gmail.com
}

\begin{abstract}
This article presents a method to create a digital twin for sauna. The digital twin deploys a data analytics method based on fuzzy similarity measure for data summarization to provide real-time actionable instructions. The intended outcome of the digital twin is enhanced safety and improved sauna experience. In this research, we collected the data through experimentation with a testbed and interviews. Our implemented digital twin simplifies the readings of nineteen sensors into a single graphical form for the user that illustrates the overall sauna conditions and assists the sauna manager with condition optimization. Results illustrated the effectiveness of the digital twin in achieving an enhanced sauna condition for the users and providing the sauna manager with a decision support system. The method of creating the digital twin described in this article has potential implications for the condition monitoring, predictive maintenance, and enhancing the safety and security of complex systems.
\end{abstract}

Index Terms-Digital twin, Internet of Things, big data analytics, smart building

\section{INTRODUCTION}

Internet of things (IoT) is now entering a new era of commercial and industrial applications as (i) the use cases become clearer [1], (ii) inexpensive and low-powered computer chips and sensors become widely available, and (iii) the network capabilities and capacities required to carry the big data created by large numbers of always-connected devices are realized (e.g., LTE-M, 5G) [2]. The potential economic impact of IoT is vast, [3], [4] and its security implications are broad [5]. One of the areas poised to immensely benefit from IoT is buildings and residential facilities [6]. Increased efficiency, improved comfort, and augmented security throughout the building life cycle are among the benefits achievable through the integration of IoT systems in the construction industry [7] and the creation of digital twins [8].

Digital twin is a concept that builds upon the advancements in the IoT systems and data analytics technologies and allows for the creation of an accurate, real-time digital simulation model for any physical system. Digital twin have applications in system behaviour analysis and what-if analysis to enhance the predictive maintenance [9]. Examples of real world implementation of digital twins can be found in aerospace, and heavy duty equipment industries.

In general, one of the main challenges faced by digital twin of systems is the real-time analysis of big data generated by numerous systems and sensors for enhanced decision making
[3], [10]. Dataset errors and noise recognition and elimination are important considerations when improving accuracy [11]. In this research, we describe in detail a methodology in the context of an experiment where the actionable data is systematically evaluated, filtered, summarized, and used to improve situation awareness for sauna users while also providing realtime suggestions for possible improvements. Moreover, the security implications of this method are discussed.

Sauna as a building service is very important in some countries. For instance in Finland, it is estimated that there are approximately two million saunas [12]. Therefore, studying saunas as spaces in buildings which are constantly under temperature and humidity variation stress is worthwhile.

Although the concept of IoT for smart buildings has been studied extensively in various contexts, the utilization of a framework for creating a digital twin for household services (e.g., saunas) to significantly simplify and enhance real-time decision-making based on sensory data needs additional study [13], [14], [15]. In this research, we contribute to filling the gap related to the creation of a real-time analytics system supporting the digital twin in interpretation of large IoT datasets. Moreover, the proposed method creates situational awareness for the users while also initiating actionable decision-support conclusions for the system manager or for service automation. The extent of this study also covers the security benefits of our proposed digital twin.

\section{Digital Twin For SMart Buildings And SAunas}

Modeling and the creation of simulations have become standard methods used to enhance system design, maintenance and testing. The resulting models and simulations play a decisive role in validating system properties and supporting design tasks. Nonetheless, early simulation-based solutions are best known for failure prediction and operation optimization [16]. The digital twin emerged in the manufacturing industry as a result of two processes. Firstly, production systems and machinery became more digitalized. Secondly, sensor networks were getting increasingly integrated. Digital twins primarily differ from using simulations in the design phase on the basis that the former includes the usage of a sensor network and physical assets, whereas the latter does not [17]. Therefore, [18] presents the following definition: "digital twins will facilitate the means to monitor, understand, 
and optimize the functions of all physical entities, living as well as nonliving, by enabling the seamless transmission of data between the physical and virtual world."

The concept of the digital twin of the building is defined by [19] as the "interaction between the real-world building's indoor environment and a digital yet realistic virtual representation model of building environment, which provides the opportunity on real-time monitoring and data acquisition." The authors of this definition envision that for indoor environments information on the lighting condition, airflow, air temperature and relative humidity $(\mathrm{RH})$ are measured, whereas the digital virtual representation indicates luminance level and computational fluid dynamics. Substantial benefits for the creation of a digital twin of a build are: 1) optimizing building services, 2) analyzing irregularities in the data, and 3) collecting, generating and creating visualizations of the environment of the building.

The application of the IoT for smart buildings is an active area of research with an extensive related body of knowledge.

\section{A. Related works}

Research has been focused on various subjects such as the comfort and efficiency of occupants [20], [7], security [21], entertainment and even medical applications [22]. Digital twin in construction, on the other hand requires further investigation. Construction industry is well-known for its slowness and reluctance in adoption of digital technologies [23], [24]. Therefore, the subject of digital twin in construction, where distinct IoT devices and sensors in combination with data analytics yield actionable information and predictions requires additional exploration to verify the potential implementation gains.

Recently, various articles and investigations presented systems for data analytics in the context of smart buildings. Researchers in [25], [26], [27], [28], and [29] have proposed frameworks for IoT data analysis. [25], explored the analytics of data for smart homes with access to cloud-based historical data. Study in [26] worked on the usage patterns of appliances in the smart homes using multidimensional patterns mining method.

Study presented in [27] illustrated the implementation of gateway-based framework for detection and configuration of new IoT appliances to enable cloud-based data analytics. A novel framework suggested by [28] introduces a new way for the adaptivity of smart home and procedures of self-learning. [30] builds upon the creation of an analytical engine for smart buildings user behavior analysis. In [29], authors propose an Hadoop ecosystem-based IoT big data analytics framework for cities to work with sensor inputs from various events and functions of a smart city. [31], and [32] deal with challenges arising from edge big data analytics such as data latency issues. These studies suggest distance reduction between the edge computing resources and data sources as a solution. However, none of the prior studies utilize fuzzy similarity based summarization for smart home decision support system.
Moreover, in the context of the smart sauna, previous research has described the potential benefit of an IoT-enabled sauna in preventing household fires [13], however, the study falls short of utilizing multi-sensor system and combing it with advanced data analytics to create a digital twin for safety. Moreover, a prior study of IoT for saunas has focused on the establishment of a sensor network in a sauna [14] but has not explored the possibilities that arise from data abstraction and real-time decision support analytics. Additional possibilities related to automation according to user preferences and security remain to be further investigated.

\section{RESEARCH METHOD}

The methodology used to conduct this research was experimentation using a testbed. A network of nineteen sensors was installed in a sauna to collect the data related to the environmental parameters. After the initial dataset analysis and selection of the relevant sensors, the data was summarized using a mathematical method. A web application was then developed to control the sauna parameters according to a user's preference. Interviews were conducted to set these preferences.

For this research we created the three main elements of a digital twin which are the IoT wireless sensor network (WSN), data integration and analytics, and 3D model components. The nineteen senors were connected to a Raspberry Pi (RPi) 3B via Bluetooth low energy (BLE) technology to create a WSN, the collected data was uploaded to the cloud every second and also stored on the RPi as a .CSV file. The data analytics method used in this research is based on the fuzzy number similarity which enables summarization of big data. The 3D model of the sauna was created in Unity software which also allows for the integration of sensor data and analytics results.

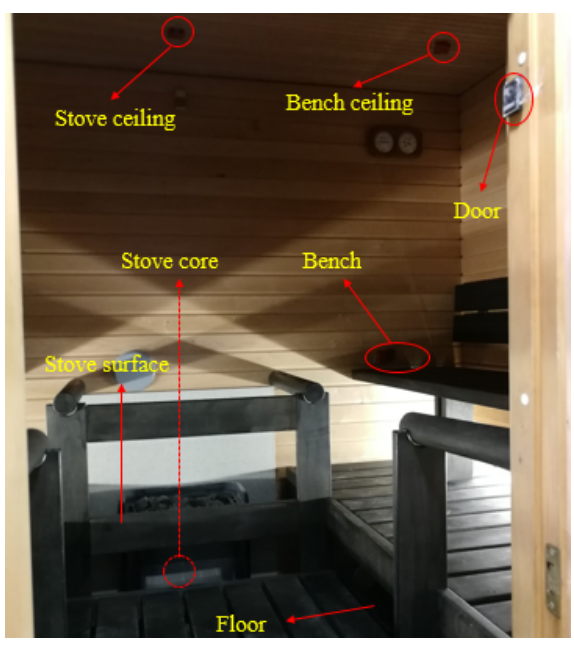

Fig. 1. Sensor configuration in the sauna for the experiment.

As Fig. 1 illustrates, the sensors of the testbed inside the sauna were installed in eight different locations, including the stove core (temperature), the stove surface (temperature), the ceiling above the stove (temperature, relative humidity), the ceiling above the bench (temperature, relative humidity), the 
TABLE II

USERS' DATA PACKAGE AND MANAGERS' DATA PACKAGE

bench seat (temperature, $\mathrm{CO}_{2}$ concentration, relative humidity, absolute humidity, moist gas energy, $\mathrm{O}_{2}$ concentration), the bench footrest (relative humidity, absolute humidity, temperature), the door (temperature, relative humidity), and the outdoors (temperature, relative humidity).

Eight sensors were selected through the analysis of the system and by determining sensors dependencies (Tables III and IV). To determine the optimal sauna conditions, 29 sauna users were interviewed (after their stay in the sauna) to collect feedback about their sauna experience, and sauna characteristics were monitored in real time. Participants were asked four main questions related to the personal qualitative evaluation of the sauna temperature (temp.), relative humidity $(\mathrm{RH})$, and air quality (AQ) as well as the estimated time of their stay in the sauna and any additional comments (Table I). The qualitative answers were quantified on a scale from 0 to 10 , with 10 being perfect feedback. The feedback for sauna temperature, humidity, and air quality were then visualized on separate timelines to facilitate their comparison to the sensor readings. This setup allowed us to realistically determine the optimal sauna parameters for our model.

TABLE I

A SAMPLE OF THE TABLE USED TO PRESENT THE INTERVIEW RESUlTS

\begin{tabular}{|c|c|c|c|c|c|c|}
\hline No. & Time & Temp. & Humidity & $A Q$ & Stay period & Comments \\
\hline 1 & $16: 20$ & Good & Good & Poor & 10 min & Near the door is cold \\
\hline
\end{tabular}

A fuzzy similarity method developed by Jaribion et al. [15], [20] was used to summarize the data from the eight selected sensors (Table II) by comparing them with the parameters of the sauna in optimal condition.

\section{A. Details of raw sensor data processing}

The measurements of the sensors contained various parameters (e.g., time, temperature, absolute humidity, relative humidity, $\mathrm{O}_{2}$ concentration, $\mathrm{CO}_{2}$ concentration, and moist gas energy). According to the work of Jaribion et al. [15], in a time series, each measured parameter can be defined as a variable and indicated by $x(i)$. The data can therefore be presented by a vector " $x$ " that consists of the number of variables " $i$." However, only some of these variables were considered when comparing the similarity of the sauna status to an optimal and preferred sauna condition. In this case, the selection of the considered parameters was made with the assistance of experts, analysis of the system, and the determination of sensor dependencies. As a result, two different data packages were used separately for the users and the manager of the sauna (facility manager) to describe the sauna conditions. The parameters that the decision maker considered for the users' data package and manager's data package are illustrated by

\begin{tabular}{|c|c|c|c|c|}
\hline $\begin{array}{c}\text { Data } \\
\text { package }\end{array}$ & Sensor & Considered parameter & Unit & Variable \\
\hline \multirow{4}{*}{ Users } & Bench & $\mathrm{O}_{2}$ concentration & $\%$ & $y(1)$ \\
\cline { 2 - 5 } & Bench & Temperature & ${ }^{\circ} \mathrm{C}$ & $y(2)$ \\
\cline { 2 - 5 } & Bench & $\mathrm{CO}_{2}$ concentration & $\mathrm{ppm}$ & $y(3)$ \\
\cline { 2 - 5 } & Floor & Temperature & ${ }^{\circ} \mathrm{C}$ & $y(4)$ \\
\hline \multirow{4}{*}{ Manager } & Stove core & Temperature & ${ }^{\circ} \mathrm{C}$ & $z(1)$ \\
\cline { 2 - 5 } & Bench & Moist gas energy & $\mathrm{kJ} / \mathrm{kg}$ & $z(2)$ \\
\cline { 2 - 5 } & Bench & Relative humidity & $\%$ & $z(3)$ \\
\cline { 2 - 5 } & Bench & $\mathrm{CO}_{2}$ concentration & $\mathrm{ppm}$ & $z(4)$ \\
\hline
\end{tabular}

vector " $y$ " and vector " $z$ ", respectively, that each of them consists of four variables (Table II).

\section{B. Data analytics and summarization}

Jaribion et al.'s data summarization method [15] was selected to analyze the data and to provide comprehensive, accurate, and actionable information. According to [15], the units must be normalized to compare the similarity of the sauna to optimal conditions. According to the decision maker, for the users' data package, the value of $0.1 \% \mathrm{O}_{2}$ concentration change was equivalent to a five-degree temperature change and a 100 ppm $\mathrm{CO}_{2}$ change. For the manager's data package, the value of a 10 degree temperature change was equivalent to a $10 \mathrm{~kJ} \backslash \mathrm{kg}$ moist gas energy change, a $1 \%$ relative humidity change, and a 100 ppm $\mathrm{CO}_{2}$ change.

Based on the four considered parameters related to each data package, " $y$ " was used to create a trapezoidal fuzzy number $\tilde{A}$, which is presented in (1) and is related to the users' data package. The variable " $z$ " was used to make a trapezoidal fuzzy number $\tilde{C}$, which is presented in (2) and is related to the managers' data package.

$$
\begin{aligned}
\tilde{A} & =\left(y(1), y(2), y(3), y(4) ; w_{\tilde{A}}\right)=\left(a_{1}, a_{2}, a_{3}, a_{4} ; w_{\tilde{A}}\right) \\
\tilde{C} & =\left(z(1), z(2), z(3), z(4) ; w_{\tilde{C}}\right)=\left(c_{1}, c_{2}, c_{3}, c_{4} ; w_{\tilde{C}}\right)
\end{aligned}
$$

Based on the results of the conducted interviews, optimal sauna parameters were set with regard to the considered parameters of the data packages (Table II) by comparing the exact time of the reports and the sensor readings at that time. For each data package and its interface, two different ideal reference point were introduced.

After normalization, the ideal reference point related to the users' data package and the manager's data package can be represented by the trapezoidal fuzzy numbers $\tilde{B}$ (3) and $\tilde{D}$ (4), respectively.

$$
\begin{aligned}
& \tilde{B}=\left(y_{o}(1), y_{o}(2), y_{o}(3), y_{o}(4) ; w_{\tilde{B}}\right)=\left(b_{1}, b_{2}, b_{3}, b_{4} ; w_{\tilde{B}}\right) \\
& \tilde{D}=\left(z_{o}(1), z_{o}(2), z_{o}(3), z_{o}(4) ; w_{\tilde{D}}\right)=\left(d_{1}, d_{2}, d_{3}, d_{4} ; w_{\tilde{D}}\right)
\end{aligned}
$$


Based on the decision maker's opinion, the heights of all fuzzy numbers $\left(w_{\tilde{A}}, w_{\tilde{B}}, w_{\tilde{C}}\right.$, and $\left.w_{\tilde{D}}\right)$ are equal to one in all calculations.

According to the results of the conducted interviews (Table I), in this case of the users' data package, the value of a $21 \% \mathrm{O}_{2}$ concentration, 70 degrees Celsius temperature for the bench, and 35 degrees Celsius temperature for the floor were optimal and preferred for sauna users. However, the level of the $\mathrm{CO}_{2}$ concentration should not exceed $800 \mathrm{ppm}$. For the manager's data package, the value of a 290 degrees Celsius temperature for the stove, $300 \mathrm{~kJ} \backslash \mathrm{kg}$ moist gas energy, and $18 \%$ relative humidity were optimal and preferred for sauna users, and the level of the $\mathrm{CO}_{2}$ concentration should not exceed $800 \mathrm{ppm}$. Moreover, these ideal reference points can be modified to meet the needs of sauna users according to their preference.

In order to compare the collected data with ideal reference points, Zuo et al.'s similarity measure [33] was utilized. The calculation process for measuring the similarity of two nonstandard fuzzy number $\tilde{A}$ and $\tilde{B}$ was described in detail by Zuo et al. [33], and a more complementary explanation was presented by Jaribion et al. [15]. However, (5) can be used to measure the similarity of $\tilde{A}$ and $\tilde{B}$ as well as $\tilde{C}$ and $\tilde{D}$ :

$$
\begin{aligned}
& S(\tilde{A}, \tilde{B})=\left(1-\frac{\sum_{i=1}^{4}\left|a_{i}-b_{i}\right|}{4}\right)\left(1-\left|x_{\tilde{A}}^{*}-x_{\tilde{B}}^{*}\right|\right) \times \\
& \frac{\min (P(\tilde{A}), P(\tilde{B}))+\min (a(\tilde{A}), a(\tilde{B}))}{\max (P(\tilde{A}), P(\tilde{B}))+\max (a(\tilde{A}), a(\tilde{B}))}
\end{aligned}
$$

After measuring the similarity between the value of considered variables and the ideal reference points of the decision maker related to sauna conditions, the acceptable range for considered variables and an appropriate time interval should be specified. Based on the range of similarity percentage, three different sauna experience can be defined for both users and manager: a perfect sauna experience, a mediocre sauna experience, and a poor sauna experience. In this way, the data can be significantly summarized, and the users and manager can identify the current sauna status and complete the required implementation to improve the sauna experience. The acceptable percentage of similarity and desired time interval depend on the acceptable data range of the decision maker, which has been determined by interviews related to the experience of sauna users. Classification of the similarity percentage of three different sauna experiences can be calculated by computing the similarity percentage of borderline points. According to the decision maker, for both users and manager, more than $85 \%$ similarity to the ideal reference point was identified as a "perfect sauna experience." A similarity of between $60 \%$ and $85 \%$ of the optimal reference point was identified as "mediocre sauna experience," and a similarity that was lower than $60 \%$ to the optimal reference point is identified as a "poor sauna experience."

\section{Development of the Web Application}

Python code was developed according to the methodology detailed in the previous section as the back-end system. Fig.
2 depicts the process flow of the web application.

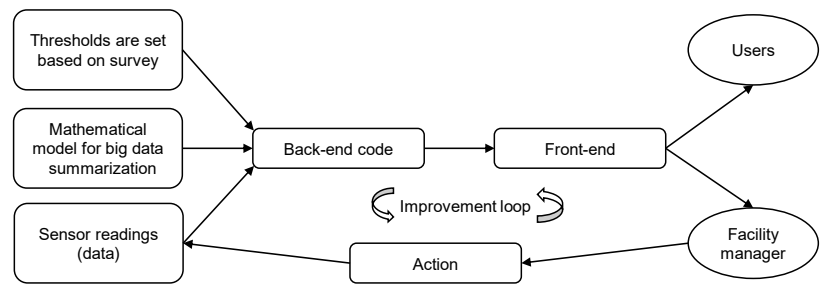

Fig. 2. The process flow of the web application.

The complete experimental setup consisted of multiple components. These components are schematically represented in Fig. 3. The sensor data follows a linear path from its source (the sensors) to the end user. The various sensors present in the sauna regularly publish their measurements to an Internetconnected database. This database enables other devices to retrieve both the most recent measurements and historical data. All data is publicly accessible and offered over a REST API. The implementation of the mathematical model is deployed separately from the business logic, in order to maintain a clear separation of concern. A serverless function running on the Google Cloud Platform is responsible for retrieving the latest data from the REST API, process it using the mathematical model, and making the results accessible to the end user. Serverless functions are self-contained pieces of code, which automatically provision their resources based on the demand, thus yielding a highly scalable solution. Lastly, the end user can access the aggregated data using a simple and easy to use responsive front-end interface.

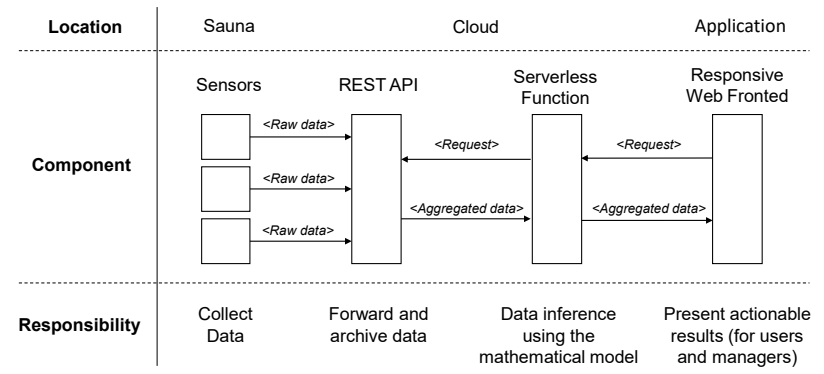

Fig. 3. Component overview of the data processing pipeline.

Discussion with experts made it apparent that the data packages of the sauna should differ based on the role of the end user. Distinct similarity measures were therefore computed based on the intent of the end user. The two roles supported by the front-end interface are the direct "Users" of the sauna and "Facility Managers," the latter who, in the case of a sauna in a spa, oversee the quality control process of several saunas.

The front-end is designed such that it offers actionable data in a simplistic view, ensuring high usability for novice users. The simplistic view consists of the similarity percentage depicted as a happy, neutral, or unhappy smiley face for similarities near the optimum, with a minor deviation from 
the optimum, or strongly diverging from the optimum, respectively. On the other hand, in the facility manager's interface, when the sauna is in a non-optimal condition, for example as a result of maloperation, the system offers suggestions for how to restore the sauna to an optimal state.

Fig. 4 presents the interface of the created digital twin of the sauna used by the manager to keep the sauna in optimal condition for the users. In this snapshot which is represented as point $\mathrm{D}$ in the Fig. 5, the sauna manager is given a suggestion by the analytics algorithm to turn on the ventilation system in order to reduce the $\mathrm{CO}_{2}$ level inside the sauna. Moreover, the digital twin (Fig. 4) provides the manager a quick summary of the sauna condition in simple graphical form complemented with the a figure that illustrates the similarity of current sauna condition to ideal. The interface also gives a real-time view of the sensor readings, sensor locations and also a root-cause evaluation for sauna condition degradation. The cause of sauna condition degradation in Fig. 4 is shown to be a high $\mathrm{CO}_{2}$ level while the readings from other sensors are inside the predefined limits. The exact reading of the $\mathrm{CO}_{2}$ sensor in this example is $1203 \mathrm{ppm}$.

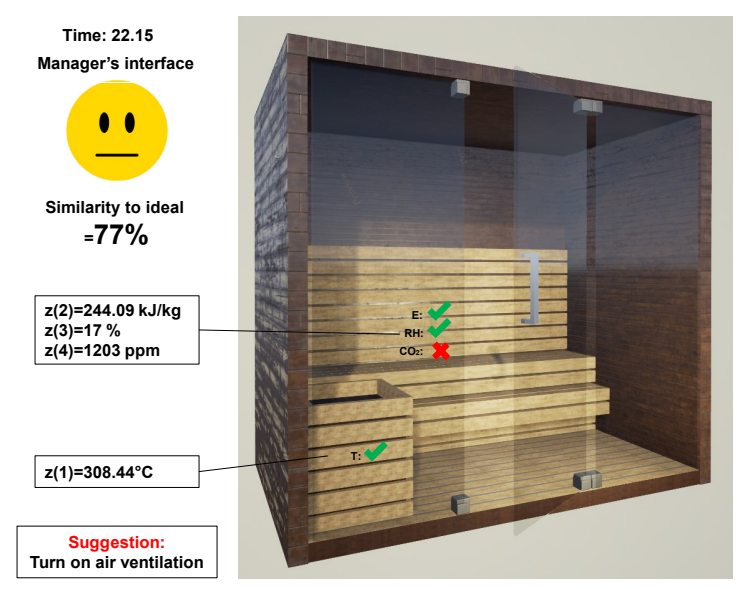

Fig. 4. Digital twin interface of sauna for the manager

Fig. 5 presents a time series showing the effectiveness of the digital twin developed in this research in action. In this time series, changes in the similarity of sauna to ideal condition over a period of 47 minutes is tracked. At point $\mathrm{A}$, the similarity value shown on the managers interface is 73 percent. The deterioration of sauna condition causes the similarity measure to fall to point $\mathrm{B}$ or 26 percent. At this time the user's similarity measure is at point $\mathrm{C}, 86$ percent which has fell from 88 percent. Manager intervenes with a corrective action at point B and the sauna condition similarity measure starts to improve on the manager interface and also the decline on the user's interface stops. The large difference between the similarity measure on manager's interface with user's interface is due to the design and sensitivity of these measures. The managers similarity measure is more sensitive to changes and is based on different sensors that are positioned to allow early warning of the condition deterioration that can effect the sauna condition for the users in a number of minutes. Therefore, with corrective actions based on the digital twin algorithm a sauna manager can keep the sauna continuously in a optimal condition for users.

\section{Discussion}

One of the clear applications of the digital twin discussed is providing the users and manager of the system with realtime abstract situational awareness, which can lead to more accurate decisions to correct the system when it deviates from predefined optimal conditions. This method depicts the most relevant environmental parameters before applying a summarization algorithm to them. This assists the system manager, as he should not deal with large sets of data collected from numerous sensors. The system manager is able to take actions that are suitable and proportionate to the system changes and potential anomalies. Such a system can control the environmental parameters of a large facility and only point out and address the issues when they take place. Another use case of the system detailed in this research is the provision of advanced system security. Based on the large historical data sets collected from the digital twin in the regular annual operation of a household sauna, a "digital ghost" can be created [34] to complement the digital twin in monitoring the system behavior and preventing anomalies caused by internal system failure. Moreover, this has application in smart connected homes where the cyber security is of utmost importance.

\section{CONCLUSIONS}

A method to create the digital twin of a sauna is presented. A web-based digital twin was developed based on a fuzzy similarity measure algorithm to provide the users with a simple real-time graphical representation of the sauna while keeping the sauna manager aware of the most important parameters to control the system. After the user has set a preference for the sauna condition involving temperature, humidity and air quality, our proposed digital twin effectively keeps the sauna conditions close to the optimal state. The output of the digital twin can be sent to the sauna manager or to an automation system for action. The implications of this method go beyond smart saunas, as the digital twin can be used to enhance condition monitoring and maintenance planning in complex systems.

\section{REFERENCES}

[1] I. Lee and K. Lee, "The internet of things (iot): Applications, investments, and challenges for enterprises," Business Horizons, vol. 58, no. 4, pp. 431-440, 2015.

[2] P. Schulz, M. Matthe, H. Klessig, M. Simsek, G. Fettweis, J. Ansari, S. A. Ashraf, B. Almeroth, J. Voigt, I. Riedel et al., "Latency critical iot applications in $5 \mathrm{~g}$ : Perspective on the design of radio interface and network architecture," IEEE Communications Magazine, vol. 55, no. 2, pp. 70-78, 2017.

[3] J. Gubbi, R. Buyya, S. Marusic, and M. Palaniswami, "Internet of things (iot): A vision, architectural elements, and future directions," Future generation computer systems, vol. 29, no. 7, pp. 1645-1660, 2013.

[4] J. Manyika, M. Chui, P. Bisson, J. Woetzel, R. Dobbs, J. Bughin, and D. Aharon, "Unlocking the potential of the internet of things," McKinsey Global Institute, 2015. 


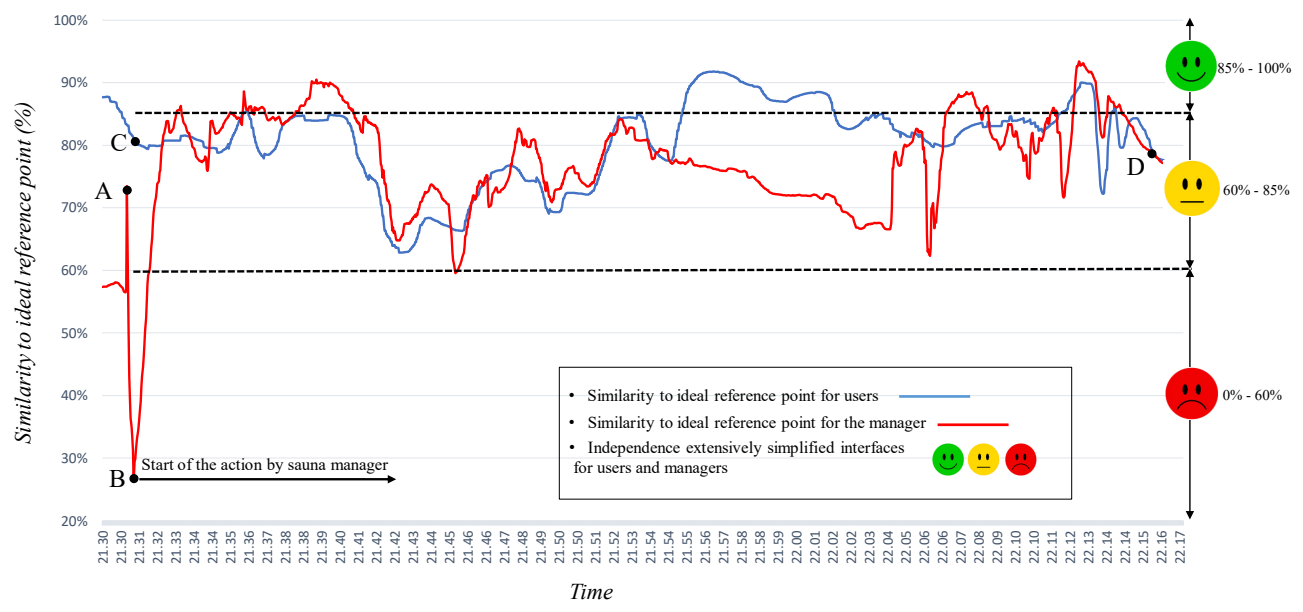

Fig. 5. The similarity of sauna condition to the ideal state over a period of 50 minutes for both users and manager.

[5] Z.-K. Zhang, M. C. Y. Cho, C.-W. Wang, C.-W. Hsu, C.-K. Chen, and S. Shieh, "Iot security: ongoing challenges and research opportunities," in 2014 IEEE 7th international conference on service-oriented computing and applications. IEEE, 2014, pp. 230-234.

[6] S. D. T. Kelly, N. K. Suryadevara, and S. C. Mukhopadhyay, "Towards the implementation of iot for environmental condition monitoring in homes," IEEE sensors journal, vol. 13, no. 10, pp. 3846-3853, 2013.

[7] N. H. Motlagh, S. H. Khajavi, A. Jaribion, and J. Holmstrom, "An iot-based automation system for older homes: a use case for lighting system," in 2018 IEEE 11th Conference on Service-Oriented Computing and Applications (SOCA). IEEE, 2018, pp. 1-6.

[8] S. H. Khajavi, N. H. Motlagh, A. Jaribion, L. C. Werner, and J. Holmström, "Digital twin: Vision, benefits, boundaries, and creation for buildings," IEEE Access, vol. 7, pp. 147 406-147 419, 2019.

[9] A. Jaribion, S. H. Khajavi, M. Öhman, A. Knapen, and J. Holmström, "A digital twin for safety and risk management: a prototype for a hydrogen high-pressure vessel," in International Conference on Design Science Research in Information System and Technology. Springer, 2020.

[10] M. M. Rathore, A. Ahmad, A. Paul, and S. Rho, "Urban planning and building smart cities based on the internet of things using big data analytics," Computer Networks, vol. 101, pp. 63-80, 2016.

[11] M. J. Khoury and J. P. Ioannidis, "Big data meets public health," Science, vol. 346, no. 6213, pp. 1054-1055, 2014.

[12] M. Norros, "Bare facts of the sauna in finland," May 2009, accessed 2020-04-26. [Online]. Available: https://finland.fi/life-society/bare-factsof-the-sauna/

[13] A. Markkanen, "Connected versus intelligent devices in the iot and in saunas," The IEEE IoT Newsletter, 2015.

[14] L. Korte et al., "Saunaxio: Sauna monitoring and remote-control platform," JAMK University of Applied Sciences, 2018.

[15] A. Jaribion, S. H. Khajavi, N. H. Motlagh, and J. Holmström, "[wip] a novel method for big data analytics and summarization based on fuzzy similarity measure," in 2018 IEEE 11th Conference on Service-Oriented Computing and Applications (SOCA). IEEE, 2018, pp. 221-226.

[16] S. Boschert, C. Heinrich, and R. Rosen, "Next generation digital twin," in Proc. TMCE. Las Palmas de Gran Canaria, Spain, 2018, pp. 209217.

[17] F. Tao, J. Cheng, Q. Qi, M. Zhang, H. Zhang, and F. Sui, "Digital twindriven product design, manufacturing and service with big data," The International Journal of Advanced Manufacturing Technology, vol. 94, no. 9-12, pp. 3563-3576, 2018.

[18] A. El Saddik, "Digital twins: The convergence of multimedia technologies," IEEE multimedia, vol. 25, no. 2, pp. 87-92, 2018.

[19] A. N. Nasaruddin, T. Ito, and T. B. Tuan, "Digital twin approach to building information management," in The Proceedings of Manufacturing Systems Division Conference 2018. The Japan Society of Mechanical Engineers, 2018, p. 304.

[20] A. Jaribion, S. H. Khajavi, and J. Holmström, "Iot-enabled workplaces: A case study of energy management and data analytics," in IECON 2019-45th Annual Conference of the IEEE Industrial Electronics Society, vol. 1. IEEE, 2019, pp. 5325-5330.

[21] A. Dorri, S. S. Kanhere, R. Jurdak, and P. Gauravaram, "Blockchain for iot security and privacy: The case study of a smart home," in 2017 IEEE international conference on pervasive computing and communications workshops (PerCom workshops). IEEE, 2017, pp. 618-623.

[22] B. Li and J. Yu, "Research and application on the smart home based on component technologies and internet of things," Procedia Engineering, vol. 15, pp. 2087-2092, 2011.

[23] R. Agarwal, S. Chandrasekaran, and M. Sridhar, "Imagining construction's digital future," McKinsey \& Company, 2016.

[24] F. Craveiroa, J. P. Duartec, H. Bartoloa, and P. J. Bartolod, "Additive manufacturing as an enabling technology for digital construction: A perspective on construction 4.0," sustainable development, vol. 4, p. 6 , 2019.

[25] H. Cai, B. Xu, L. Jiang, and A. V. Vasilakos, "Iot-based big data storage systems in cloud computing: perspectives and challenges," IEEE Internet of Things Journal, vol. 4, no. 1, pp. 75-87, 2016.

[26] G. Poghosyan, I. Pefkianakis, P. Le Guyadec, and V. Christophides, "Extracting usage patterns of home iot devices," in 2017 IEEE Symposium on Computers and Communications (ISCC). IEEE, 2017, pp. 13181324.

[27] B. Kang, D. Kim, and H. Choo, "Internet of everything: A large-scale autonomic iot gateway," IEEE Transactions on Multi-Scale Computing Systems, vol. 3, no. 3, pp. 206-214, 2017.

[28] M. Sultan and K. N. Ahmed, "Slash: Self-learning and adaptive smart home framework by integrating iot with big data analytics," in 2017 Computing Conference. IEEE, 2017, pp. 530-538.

[29] M. M. Rathore, A. Ahmad, and A. Paul, "Iot-based smart city development using big data analytical approach," in 2016 IEEE international conference on automatica (ICA-ACCA). IEEE, 2016, pp. 1-8.

[30] S. Hossain and G. Muhammad, "Emotion-aware connected healthcare big data towards 5g," IEEE Internet of Things Journal, vol. 5, no. 4, pp. 2399-2406, 2017.

[31] J. He, J. Wei, K. Chen, Z. Tang, Y. Zhou, and Y. Zhang, "Multitier fog computing with large-scale iot data analytics for smart cities," IEEE Internet of Things Journal, vol. 5, no. 2, pp. 677-686, 2017.

[32] N. M. Gonzalez, W. A. Goya, R. de Fatima Pereira, K. Langona, E. A. Silva, T. C. M. de Brito Carvalho, C. C. Miers, J.-E. Mångs, and A. Sefidcon, "Fog computing: Data analytics and cloud distributed processing on the network edges," in 2016 35th International Conference of the Chilean Computer Science Society (SCCC). IEEE, 2016, pp. 1-9.

[33] X. Zuo, L. Wang, and Y. Yue, "A new similarity measure of generalized trapezoidal fuzzy numbers and its application on rotor fault diagnosis," Mathematical Problems in Engineering, vol. 2013, 2013.

[34] T. Alhart, "Night watch 2.0: Meet the digital ghost in the machine," Oct. 2017, accessed 2019-06-17. [Online]. Available: https://www.ge.com/reports/night-watch-2-0-meet-digital-ghostmachine/ 